\title{
Design of Advanced Power Distribution Networks Including DGs and D-FACT Devices
}

\author{
Deepak Kumar ${ }^{1} \cdot$ S. R. Samantaray ${ }^{1}$
}

Received: 25 April 2016/ Accepted: 6 May 2016/Published online: 27 May 2016

(C) Indian National Academy of Engineering 2016

\begin{abstract}
This paper presents an application of a multiobjective seeker-optimization-algorithm (MOSOA) for solving multi-objective design of advanced power distribution systems (PDS) considering fixed cost, non-linear variable cost and reliability as the objective functions including DGs (distributed generations) and D-FACT (distribution-FACT) devices. The proposed planning methodology uses contingency-load-loss index (CLLI) for reliability evaluation, which is independent of failure rate and fault repair duration of the feeder branches. To enhance the reliability and efficiency of existing PDS, planning strategy includes distribution automation devices such as automatic recloser (RA). Performance of the proposed approach is assessed and illustrated on a 54-bus PDS, considering real-time design practices. Furthermore, a qualitative comparison is made with NSGA-II, showing the efficacy of the proposed planning approach.
\end{abstract}

Keywords Multi-objective seeker-optimizationalgorithm $\cdot$ Non-dominated sorting genetic algorithm-II (NSGA-II) · Power distribution system design . Contingency-load-loss index (CLLI)

\section{Introduction}

Design of an optimal power distribution system (PDS) involves simultaneous optimization of frequently competing multiple objectives such as minimization of the

S. R. Samantaray

sbh_samant@yahoo.co.in

1 School of Electrical Sciences, Indian Institute of Technology, Bhubaneswar, India installation cost of the new facilities such as substation, distributed generations (DGs), automatic protective devices such as RAs, D-FACT (distribution-FACTs devices such as D-STATCOM: distribution-static compensator), feeders, etc., minimization of operational costs such as energy loss cost and maximization of system reliability to design a cost-effective and reliable network (El-Kady 1984; Samui et al. 2012; Kumar et al. 2014a, b; Najafi et al. 2009; ; Rosado and Agustín 2006; Mendoza et al. 2006; Carrano et al. 2006; Ramírez-Rosado and Domínguez-Navarro 2004; Ganguly et al. 2012; Kumar et al. 2015a, b; Nekooei et al. 2013; Kumar and Samantaray 2014). Research work based on numerical based methods such as "Mixed integer linear programming approach" (El-Kady 1984), the "Direct search approach" (Samui et al. 2012) etc. for PDS planning have limited scope in practical applications as almost all the practical problems involve objective functions that are non-linear, non-convex, and non-differentiable in nature. In this regard, heuristic-based methods (Najafi et al. 2009; Kumar et al. 2014b) have distinct advantages such that it can handle complex problems and also it do not require any gradient information. Several multi-objective evolutionary algorithms have been applied and found suitable for PDS planning problems (Rosado and Agustín 2006; Mendoza et al. 2006; Carrano et al. 2006; Ramírez-Rosado and Domínguez-Navarro 2004; Ganguly et al. 2012). Most of the literature (Rosado and Agustín 2006; Mendoza et al. 2006; Carrano et al. 2006; RamírezRosado and Domínguez-Navarro 2004) have used expected energy not served (EENS) for reliability estimation which is based on average failure rates and the repair durations of all the feeder branches, that is very difficult to estimate in practice as the occurrence of faults take place because of various unpredictable non-technical reasons. To alleviate such problems, PDS planning based on a reliability index 
(contingency-load-loss index (CLLI)) (Ganguly et al. 2012; Kumar et al. 2015a, b) is proposed, which is independent of the estimation of the failure and repair rate of the branches. In reference (Kumar et al. 2015a) and (Kumar et al. 2015 b), authors have not considered the impact of simultaneous placement of DGs and D-FACT devices on the overall PDS planning.

This paper includes the simultaneous optimal placement of DGs and D-STATCOM while designing an advanced PDS. The paper is organized as follows: problem formulation (Sect. 1), modeling of D-STATCOM (Sect. 3), description of proposed MOSOA (Sect. 4), results and discussion (Sect. 5), and the conclusions (Sect. 6).

\section{Problem Formulation}

In this paper in order to carry out the optimal MO design of PDS, the vectors of the objective function to be minimized is $u=[u 1, u 2]$, where $u 1$ is the objective function of global economic cost and $u 2$ is the objective function related to the reliability of the PDS.

$u 1=C_{\text {System }}=C_{\text {Fixed }}+C_{\text {Variable }}+C_{\text {Maintenance }}$

where,

$$
\begin{aligned}
C_{\text {Fixed }}= & \sum_{k \in N_{S}} \sum_{b \in N_{b}}\left[\left(C_{k}^{F}\right)_{b}\left(Y_{k}\right)_{b}\right]+\sum_{D \in N_{D}} \sum_{g \in N_{g}}\left[\left(C_{D}^{F}\right)_{g}\left(Y_{D}\right)_{g}\right] \\
& +\sum_{k \in N_{D F}} \sum_{f \in N_{f}}\left[\left(C_{D F}^{F}\right)_{f}\left(Y_{D F}\right)_{f}\right]+\sum_{(i, j) \in N_{F}} \sum_{a \in N_{a}}\left[\left(C_{i, j}^{F}\right)_{a}\left(Y_{i, j}\right)_{a}\right] \\
& +\sum_{(i, j) \in N_{F}} \sum_{m \in N_{m}}\left[\left(C_{i, j}^{F}\right)_{m}\left(Y_{i, j}\right)_{m}\right]+\sum_{(i, j) \in N_{F E}} \sum_{a \in N_{a}}\left[\left[\left(C_{i, j}^{F}\right)_{E}\right]_{a}\left(Y_{i, j}\right)_{a}\right] \\
& +\sum_{k \in N_{S E}} \sum_{b \in N_{b}}\left[\left[\left(C_{k}^{F}\right)_{E}\right]_{b}\left(Y_{k}\right)_{b}\right] \\
C_{\text {Variable }}= & \sum_{k \in N_{S}} \sum_{b \in N_{b}}\left[\left(C_{k}^{V}\right)_{b}\left(X_{k}\right)_{b}^{2}\right] t_{a}+\sum_{D \in N_{D}} \sum_{g \in N_{g}}\left[\left(C_{D}^{V}\right)_{g}\left(X_{D}\right)_{g}^{2}\right] t_{a} \\
& +\sum_{(i, j) \in N_{F}} \sum_{a \in N_{a}}\left[\left(C_{i, j}^{V}\right)_{a}\left\{\left(X_{i, j}\right)_{a}^{2}+\left(X_{j, i}\right)_{a}^{2}\right\}\right] t_{a} \\
& +\sum_{(i, j) \in N_{F E}}\left[\left(C_{i, j}^{V}\right)_{E}\left\{\left(X_{i, j}\right)_{E}^{2}+\left(X_{j, i}\right)_{E}^{2}\right\}\right] t_{a}+\sum_{k \in N_{a}}\left(C_{k}^{V}\right)_{E}\left(X_{k}\right)_{E}^{2} t_{a} \\
C_{\text {Maintenance }}= & \sum_{(i, j) \in N_{F}} \sum_{a \in N_{a}}\left[\left(C_{i, j}^{M}\right)\right]_{a} t_{a}+\sum_{(i, j) \in N_{F E}} \sum_{a \in N_{a}}\left[\left(C_{i, j}^{M}\right)_{E}\right]_{a} t_{a} \\
& +\sum_{k \in N_{S}} \sum_{b \in N_{b}}\left[\left(C_{k}^{M}\right)_{b}\left(Y_{k}\right)_{b}\right] t_{a}+\sum_{k \in N_{S E}} \sum_{b \in N_{b}}\left[\left[\left(C_{k}^{M}\right)_{E}\right]_{b}\left(Y_{k}\right)_{b}\right] t_{a}
\end{aligned}
$$

where, $N F$ Set of proposed feeder routes to be built; NS Set of nodes associated with proposed locations for building substations; $N_{D F}$ Set of nodes associated with selected locations for building DSTATCOMs; $N_{D}$ Set of nodes associated with proposed locations for building DGs; $N_{b}\left(N_{m}\right)$ Set of proposed substa. (RA/CB) sizes to be built; $N g(N a)$ Set of proposed DG (feeder) sizes to be built; $(X i, j) a$ Power flow in $\mathrm{kVA}$, carried though route $(i, j) \in N F$ associated with a feeder of size $a$. $(X k) \mathrm{b}, \mathrm{E}$ Power flow in kVA, supplied froma proposed substation of size $b$ or with an existing $(E)$ one. $\left(X_{D}\right)_{g}$ Power flow in
kVA, supplied from $D \in N_{D}$ associated with a proposed DG of size $g . C^{V(F)}$ Variable (fixed) cost; $C^{M}$ Maintenance $\operatorname{cost} t_{a}$ Analysis time in years. $(Y i, j) a$ is equal to 1 if the feeder of size $a$ associated with route $(i, j) \in N F P$ is built. Otherwise, it is zero. $\left(Y_{k}\right)_{b}$ is equal to 1 if the substation of size $b$ associated with node $k \in N S P$ is built. Otherwise, it is zero. $\left(Y_{D}\right)_{g}$ is equal to 1 , if the DG with size $g$ associated with node $D \in N_{D}$ is built. Otherwise, it is zero. $\left(Y_{D F}\right)_{f}$ is equal to 1 , if the DSTATCOM with size $f$ associated with node $k \in N_{D F}$ is built. Otherwise, it is zero.

The objective function in (1) aims to minimize the investment cost i.e., $C_{\text {Fixed }}$ where, the first five terms represents the asset cost of reinforcing/constructing substations, deploying DGs, D-STATCOM, building feeders, and reinforcing RAs and circuit breakers (CBs) respectively. The sixth and seventh terms show the asset cost associated with existing feeders of size $a$ and with existing substations of size $b$, respectively.

For variable cost component $\left(C_{\text {Variable }}\right)$, the first two terms represents variable cost associated with substation and DGs respectively, which mainly considers the cost associated with the fuel used. The third, fourth and fifth terms is the variable cost associated with (proposed/existing) feeders of size $a$ (it accounts for energy loss of the system) and with existing substations of size $b$ (fuel cost), respectively. $C_{\text {Maintenance, }}$ it represents the maintenance cost per annum involved with feeders (proposed/existing), DGs and the substations (proposed/existing).

The proposed PDS planning includes a CLLI-based reliability index which can be defined as the ratio of the average of non-delivered load due to failure of all branches, taken one at a time, to the total load. Thus the objective function $u 2$ can be mathematically expressed as,

$u 2=\mathrm{CLLI}=\frac{\mathrm{NDL}_{\mathrm{Avg}} \cdot L_{\mathrm{Total}}}{=} \frac{\sum_{i=1}^{N_{F}} N D L_{i} / N_{F}}{L_{\text {Total }}}$

where,NDL ${ }_{i}$ Non-delivered load due to fault in branch

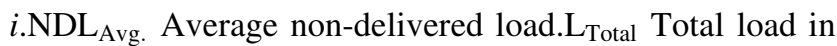
kVA.

The evaluation of CLLI index is given in detail in (Kumar and Samantaray 2014). The multi-objective problem can be formulated as:-

$\operatorname{Min} F=[$ CSystem, CLLI $]$

The simultaneous minimization of two objective functions is subjected to some technical constraints, namely:

(a) Kirchhoff's current law constraints for all nodes of the distribution network.

(b) The capacity constraint limits of the feeders and substation.

(c) The voltage level in load buses. 
(d) Network connectivity and radiality constraint of the active network $(N B=N N-1$, where $N B$ is the number of branches and $N N$ is the number of nodes).

\section{Modeling of D-STATCOM}

A D-STATCOM is represented by a synchronous voltage source and its shunt connected transformer. The equivalent corresponds to the Thevenin equivalent as seen from bus $k$, with the voltage source $E$ being the fundamental frequency component of the synchronous condenser output voltage is shown in Fig. 1. The power flow equations for the DSTATCOM are derived from first principles and assuming the following voltage source representation (Acha et al. 2004):

$E_{v R}=V_{v R}\left(\cos \delta_{v R}+\mathrm{j} \sin \delta_{v R}\right)$

Based on the shunt connection shown in Fig. 1, the net apparent, active and reactive power equations for the converter and bus $k$, can be expressed as (Acha et al. 2004):

$$
\begin{aligned}
S_{v R}= & V_{v R} I_{v R}^{*}=V_{v R} Y_{v R}^{*}\left(V_{v R}^{*}-V_{k}^{*}\right) \\
P_{v R}= & V_{v R}^{2} G_{v R}+V_{v R} V_{k}\left[G_{v R} \cos \left(\delta_{v R}-\theta_{k}\right)\right. \\
& \left.+B_{v R} \sin \left(\delta_{v R}-\theta_{k}\right)\right] \\
Q_{v R}= & -V_{v R}^{2} B_{v R}+V_{v R} V_{k}\left[G_{v R} \sin \left(\delta_{v R}-\theta_{k}\right)\right. \\
& \left.-B_{v R} \cos \left(\delta_{v R}-\theta_{k}\right)\right] \\
P_{k}= & V_{k}^{2} G_{v R}+V_{k} V_{v R}\left[G_{v R} \cos \left(\theta_{k}-\delta_{v R}\right)\right. \\
& \left.+B_{v R} \sin \left(\theta_{k}-\delta_{v R}\right)\right] \\
Q_{k}= & -V_{k}^{2} B_{v R}+V_{k} V_{v R}\left[G_{v R} \sin \left(\theta_{k}-\delta_{v R}\right)\right. \\
& \left.-B_{v R} \cos \left(\theta_{k}-\delta_{v R}\right)\right]
\end{aligned}
$$

All the parameter details and using these power equations, the linearised D-STATCOM model are given in (Acha et al. 2004).

\section{Development of Multi-Objective Seeker Optimization Algorithm}

The step-by-step solution procedure (steps 1 to 9) which shows that SOA is a simple and straight-forward approach to implement for distribution system planning problem. The details of SOA are shown in (Kumar and Samantaray 2014, Kumar et al. 2015a).

Step 1: Initialize the initial set of seekers of size $\mathrm{N}_{\mathrm{IS}}$ using fundamental loop generator that preserve the radiality operation (Kumar and Samantaray 2014) where, each seeker represents one radial configuration of dimension $\mathrm{P}$,

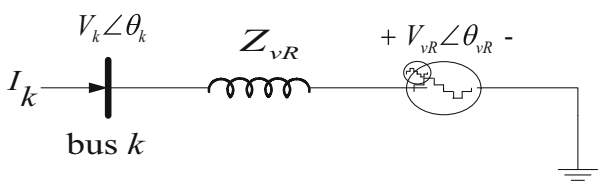

Fig. 1 DSTATCOM connected via a shunt connected transformer

which restricts the initial population that is further improved by SOA.

Step 2: In order to add a social component for the sharing of information, a neighborhood is defined for each of the $\underline{N}_{N D}$ seekers. Split the population of size $\underline{N}$ ND into $\underline{N} S P$ different sub-populations. Each sub-population is the same size.

Step 3: Evaluate the fitness value of each seeker (Kumar et al. 2014a).

Step 4: Determine best personal, global and local positions.

Step 5: Calculate different components of the search direction.

Step 6: Update the position of each seeker.

Step 7: Perform a seeker mutation using the inter-subpopulation learning scheme.

Step 8: Perform "seeker movement" operation.

Step 9: Now select the best seekers of size $\underline{N}$ ND from the set of combined solutions ("seeker mutation" and "seeker movement" operation) using a fast non-dominated sorting algorithm for the new population $\mathrm{X}_{\mathrm{t}+1}$.

Step 10: Combine $\mathrm{X}_{\mathrm{t}+1}$ (current solution) and $\mathrm{Y}_{\mathrm{t}+1}$ (previous solution) of size $\underline{N}$ ND respectively, perform fast non-dominated sorting algorithm and obtain the best set of seekers of size $\underline{N}$ ND and repeat the same procedure from step 2 for the next set of populations until the stop criterion is satisfied (either a maximum number of iterations is reached orthe objective function does not improve).

\section{Results and Discussion}

A system of 54 nodes with a proposed substation capacity of 4 MVA and total load demand of 2.032 MVA is considered to illustrate the performance of MOSOA and NSGA-II. All the details of the load point, CB data are given in (Kumar and Samantaray 2014). A backward/forward load flow algorithm of the proposed work (Samui et al. 2012) is developed on a MATLAB-R2010a platform for radial load flow analysis. A maximum of six RA's, three DG units (maximum size of DG is considered to be 
$2 \mathrm{MW}$ ), and two D-STATCOMs with capacity of \pm 1 MVAR are used in the network to enhance the overall system performance index (such as CLLI Index, voltage profile etc.). The test system is taken from ref. (Kumar et al. 2015a). The substation installation and maintenance cost (per annum) is considered to be $\$ 7,50,000$ and $\$ 10,000$ respectively. The installation cost of each DG, D-STATCOM and RA are considered to be $\$ 110,769.23, \$ 50 / \mathrm{kVAR}$ and $\$ 15,384.61$, respectively. Short circuit capacity of each RA is considered to be 20kA. The cost of operation of each substation and DG are $0.001 \$ /(\mathrm{kVA})^{2} \mathrm{~h}$. The variable costcoefficient for each line section is $0.005 \$ /(\mathrm{kVA})^{2} \mathrm{~h}$.

The abstract of the parameters used for the methodologies (MOSOA and NSGA-II) are depicted in Table 1. The complete study is carried out on a desktop PC with an Intel core i5-2400, $3.10 \mathrm{GHz}, 8 \mathrm{~GB}$ of RAM, and a 32-bit operating system using a MATLAB-R2010a platform. In this paper "Max-min" approach is applied to select the final optimal solution. In order to select the best multiobjective planning solution, the value of the objective functions are normalized using the following expression:

$$
\left(\frac{C_{\max }-C_{k}}{C_{\max }-C_{\min }}, \frac{C_{\text {LLImax }}-C_{\text {LLIk }}}{C_{\text {LLImax }}-C_{\text {LLImin }}}\right)
$$

Where, $\left(\mathrm{C}_{\max }, \mathrm{C}_{\min }, \mathrm{Ck}\right)$ and $\left(\mathrm{CLL}_{\text {Imax }}, \mathrm{CLL}_{\text {Imin }}, \mathrm{CLLI} \mathrm{k}\right)$ are the (maximum, minimum, and the value of the $\mathrm{k}$-th Pareto point) of cost and reliability function respectively. Afterward, a "Max-min" approach shown in Ganguly et al. 2012 is applied to select the best multi-objective planning solution. At first, we have evaluated the membership function values of each of the non-dominated solution in the range of $(0,1)$. Then the summation of the membership values of all the objectives of each of the non-dominated solution indicates that how well this solution satisfies all the objective functions. Afterward, the solution which is having a higher value will be selected as the most suitable solution over the Pareto-front. At the start 100 solutions have been obtained from fundamental loop generator (initial seekers), which satisfy all the system constraints. These initial set of seekers is then further modified by modifying their variables using MOSOA, defined in

Table 1 Parameters used for different techniques

\begin{tabular}{lll}
\hline Parameters & MOSOA & NSGA-II \\
\hline Population size & 100 & 100 \\
$\left(\mu_{\max }, \mu_{\min }\right)$ & $(0.95,0.0111)$ & - \\
$\left(w_{\max }, w_{\min }\right)$ & $(0.1,0.9)$ & - \\
Crossover probability & - & 0.8 \\
Mutation probability & - & 0.02 \\
Max Gen & 100 & 100 \\
Independent run & 50 & 50 \\
\hline
\end{tabular}

section IV. Pareto optimal fronts obtained by MOSOA and NSGA-II are shown in Fig. 2.

While comparing the performance with NSGA-II (Table 2), it is observed that network B has a total cost of $\$ 3,381,000$, which is $2.58 \%$ higher than A (Fig. 3). It is also found that the CLLI of B is 0.115 which is $3.60 \%$ higher than A. For the most suitable solution obtained (Network A) with the proposed approach considering DGs and D-STATCOMs, the optimal power flow result indicates that the optimal locations of DGs are at buses 34, 50 and 18 with the optimal sizes of $2.043 \mathrm{~kW}$ (Bus 34), $14 \mathrm{~kW}$ (Bus 50), and $4 \mathrm{~kW}$ (Bus 18) respectively. DSTATCOM generates $-20.22 \mathrm{kVAR}$ (Bus 52) and 18.24 kVAR (Bus 34) in order to keep the voltage magnitude within permissible limit of (0.95 to 1.05 p.u) at all the buses. The D-STATCOM parameters associated with this amount of reactive power generation are $V_{v R}=1.0018$ p.u. and $\delta_{v R}=0.001$ (Bus 52), and $V_{v R}=1.0002$ p.u. and $\delta_{v R}=0.009$ (Bus-34) respectively.

$\max \left\{\min k\left(\frac{C_{\max }-C_{k}}{C_{\max }-C_{\min }}, \frac{C_{\text {LLImax }}-C_{\text {LLIk }}}{C_{\text {LLImax }}-C_{\text {LLImin }}}\right)\right\}$

Furthermore the substation reduces its active and reactive power generation by almost 1 and $4 \%$ compared with, without DG and D-STATCOM case. In general, more reactive power is available in the network than without D-STATCOM case, and the substation connected at Bus 1 increases its share of reactive power absorption compared with without D-STATCOM case. As expected, active power flows are only marginally affected by the

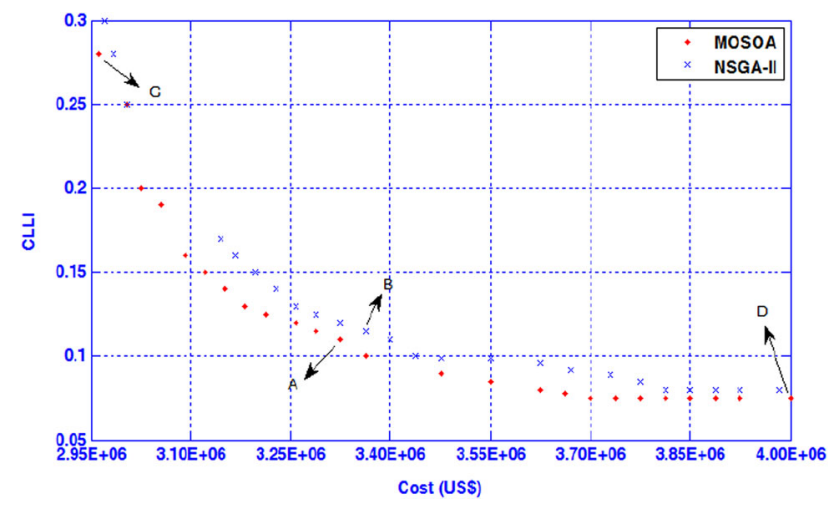

Fig. 2 Pareto front of MOSOA and NSGA-II for 54-bus system

Table 2 Comparison of most suitable solution with different techniques

\begin{tabular}{lll}
\hline Parameters & MOSOA & NSGA-II \\
\hline Cost (US\$) & $3.296 * \mathrm{E}+06$ & $3.381 * \mathrm{E}+06$ \\
CLLI & 0.1112 & 0.1150 \\
Worst voltage (p.u) & 0.9995 & 0.9993 \\
\hline
\end{tabular}


Fig. 3 Most suitable solution obtained using MOSOA: network A

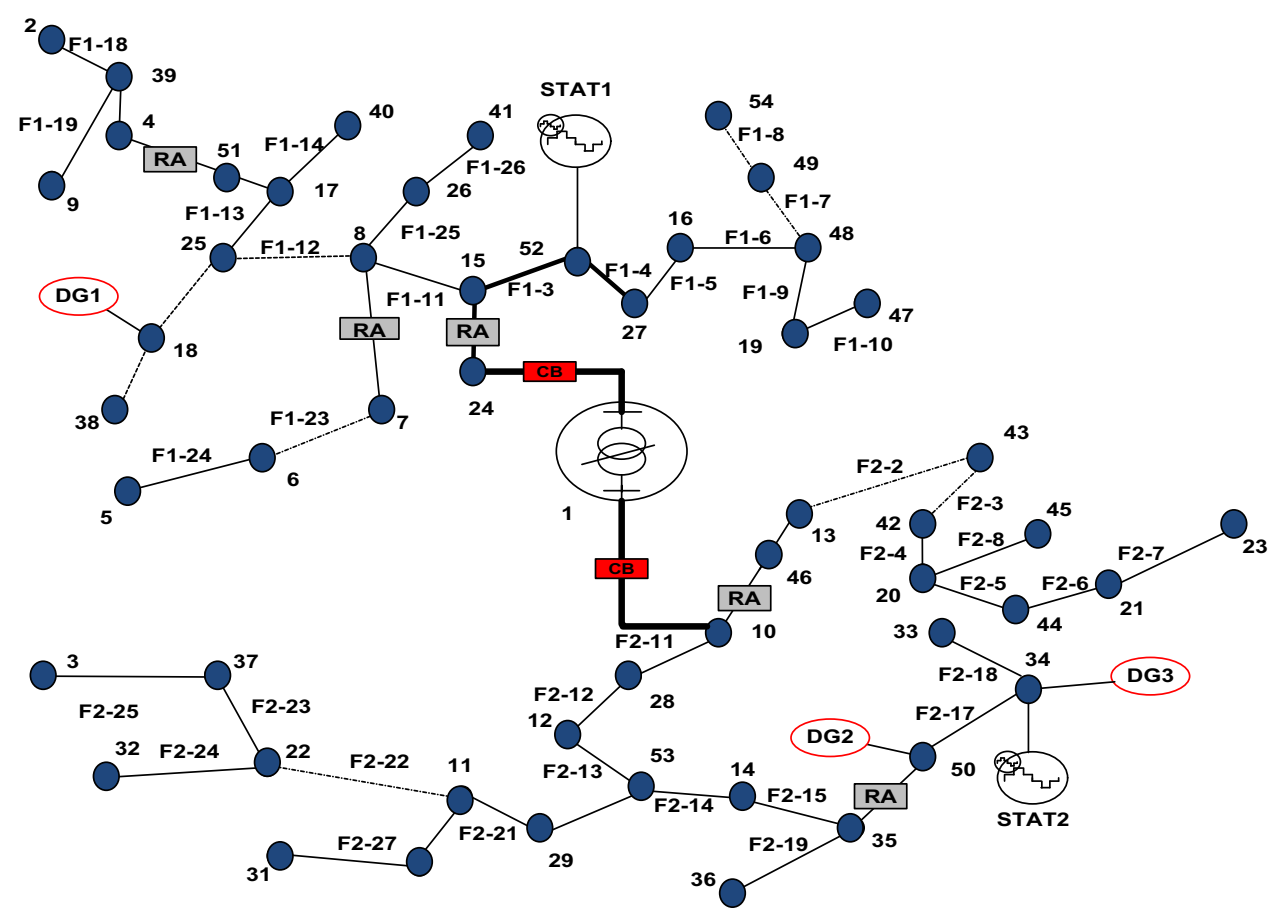

Table 3 Comparison of computational complexity

\begin{tabular}{llllllll}
\hline System & MOSOA & & & \multicolumn{2}{l}{ NSGA-II } & \\
\cline { 2 - 3 } & Shortest time $(\mathrm{s})$ & Longest time $(\mathrm{s})$ & Ave. time $(\mathrm{s})$ & & Shortest time $(\mathrm{s})$ & Longest time $(\mathrm{s})$ & Ave. time $(\mathrm{s})$ \\
\hline 54-bus & 4980.65 & 6180.56 & 5600 & 7949 & 8269 & 8025 \\
\hline
\end{tabular}

D-STATCOM installation. The Pareto-front shown in Fig. 2 clearly shows that MOSOA dominates the NSGA-II reflecting the superiority of the proposed algorithm and is computationally less intensive than NSGA-II (Table 3).

\section{Conclusion}

An advanced PDS, in which primary feeders operate in a radial configuration, has been explored in this paper using the MOSOA, which considers simultaneous optimization of the total system economic cost and overall system reliability. Extensive tests are carried out in order to find the efficacy of the proposed algorithm to design an advanced PDS including distribution automation devices, DGs and D-STATCOMs, which generates the distribution networks based on a trade-off between cost and reliability. Also, a "Max-min" approach is employed to automatically select the most suitable solution over the Pareto-front. The Pareto-front, results indicate that the MOSOA has a superior performance than that of NSGA-II, which furthermore is computationally intensive. Nevertheless, the method presented in this paper can be effective and helpful to system planners for obtaining typical designs of an advanced power distribution system.

Acknowledgments This work is fully supported by Inspire programme (DST/INSPIRE Fellowship/2012/224), DST, Ministry of science and technology, GOI, New Delhi, India.

\section{References}

Acha E, Fuerte-Esquivel CR, Ambriz-Pérez H, Angeles-Camacho C (2004) FACTS: modelling and simulation in power networks, 1st edn. Wiley, Chichester

Carrano EG, Soares LE, Takahashi RC, Saldanha RR, Neto OM (2006) Electric distribution network multiobjective design using a problem-specific genetic algorithm. IEEE Trans Power Deliv 21(2):995-1005

El-Kady MA (1984) Computer-aided planning of distribution substation and primary feeders. IEEE Trans Power App Sys PAS 103(6):1183-1189

Ganguly S, Sahoo NC, Das D (2012) Multi-objective planning of electrical distribution systems incorporating sectionalizing switches and tie-lines using particle swarm optimization. Swarm Evol Comput 3:15-32

Kumar D, Samantaray SR (2014) Design of an advanced electric power distribution systems using seeker optimization algorithm. Int J Electr Power Energy Syst 63:196-217 
Kumar D, Samantaray SR, Joos G (2014a) A reliability assessment based graph theoretical approach for feeder routing in power distribution networks including distributed generations. Int $\mathrm{J}$ Electr Power Energy Syst 57:11-30

Kumar D, Samantaray SR, Kamwa I, Sahoo NC (2014b) Reliabilityconstrained optimal placement of multiple distributed generators in power distribution network using cat-swarm-optimization. Electr Power Comp Syst 42(2):149-164

Kumar D, Samantaray SR, Kamwa I (2015a) Multi-objective design of advanced power distribution networks using restricted-population based mosoa and fuzzy-operator. IET Gen Trans Distrib 9(11):1195-1215

Kumar D, Samantaray SR, Kamwa I (2015b) MOSOA based multiobjective design of power distribution systems. IEEE Syst J (early access)

Mendoza F, Agustin JB, Dominguez-Navarro JA (2006) NSGA and SPEA applied to multi-objective design of power distribution systems. IEEE Trans Power Syst 21(4):1938-1945
Najafi S, Hosseinian SH, Abedi M, Vahidnia A, Abachezadeh S (2009) A framework for optimal planning in large distribution networks. IEEE Trans Power Syst 24(2):1019-1028

Nekooei K, Farsangi MM, Pour HN, Lee KY (2013) An improved multi-objective harmony search for optimal placement of DGs in distribution systems. IEEE Trans Smart Grid 4(1):557-567

Ramírez-Rosado IJ, Domínguez-Navarro JA (2004) Possibilistic model based on fuzzy sets for the multi-objective optimal planning of electric power distribution networks. IEEE Trans Power Syst 19(4):1801-1810

Rosado IJR, Agustín JLB (2006) New multi-objective Tabu Search algo. for fuzzy optimal planning of power distr. system. IEEE Trans Power Syst 21(1):224-233

Samui A, Singh S, Ghose T, Samantaray SR (2012) A direct approach to optimal feeder routing for radial distribution system. IEEE Trans Power Deliv 27(1):253-260 